\title{
Adaptaciones de puestos de trabajo de centros de salud en trabajadores especialmente sensibles a riesgos laborales
}

\author{
Workplace accommodations for workers especially sensitive to occupational \\ risks of community health centers
}

\section{Padilla Fortes, Ana '; Gámez de la Hoz, Joaquín²}

1. Unidad de Prevención de Riesgos y Salud Laboral. Distrito Sanitario Málaga. Servicio Andaluz de Salud. España.

2. Servicio de Salud Pública. Distrito Sanitario Costa del Sol. Málaga. Servicio Andaluz de Salud. España.

Recibido: 04-02-14

Aceptado: 06-06-14

\section{Correspondencia}

Ana Padilla Fortes

CL. Virgen Bien Aparecida 17

29007 Málaga. España.

Teléfono: 665921907

Correo electrónico: anpafo4@andaluciajunta.es

\section{Resumen}

Este estudio explora los ajustes trabajo-persona realizados en trabajadores considerados especialmente sensibles a determinados riesgos laborales en el sector sanitario. El propósito de esta investigación fue ayudar a caracterizar las adaptaciones del puesto de trabajo con motivo de la comunicación de algún tipo de discapacidad o limitación de salud que interfiere en el desempeño de tareas. A lo largo del año 2012, en los centros de salud de un distrito de atención primaria se contabilizó un total de 46 trabajadores calificados especialmente sensibles que recibieron un promedio de 3,2 medidas de adaptación en el lugar y/o puesto de trabajo. Los hallazgos mostraron una amplia variedad de ajustes que incluyeron modificaciones en la organización del trabajo, en la forma de desempeñar las tareas, y en menor grado, cambios en los factores ambientales próximos al puesto y/o lugar de trabajo. Todas las medidas estuvieron encaminadas a facilitar la continuidad de la vida laboral dentro de unos límites compatibles con la seguridad y salud del trabajador. Nuestros resultados ofrecen una primera aproximación al conocimiento de los aspectos de gestión de las limitaciones de salud que menoscaban la capacidad de los trabajadores para responder a las demandas de trabajo.

Med Segur Trab (Internet) 2014; 60 (236) 497-507

Palabras clave: Salud laboral, Adaptaciones, Discapacidad, Lugar de trabajo, Seguridad, Factor de riesgo. (Fuente: $\mathrm{MeSH}, \mathrm{NLM}$ )

Abstract

An overview was conducted of work-person adjustments engaged in workers classified as especially sensitive for certain occupational risks in health sector. The purpose of this research was to provide workplace accommodations featuring because of some disability or health limitation associated with impairments from the tasks performance. During 2012 in the community health centers of a primary care district, 46 workers were catalogued especially sensitive that received an average of 3,2 adjustment measure in workplace. The 
findings showed a wide variety of accommodations including modifications in working arrangement, in the way to performance of the tasks, and in a lesser extent, changes in near environmental factors to the workplace and/or workstation. All prevention efforts were setting on provide to maintain continuity of working life inside compatible-limits with health and safety of workers. Our results offer a first approach to the knowledge of health limitations management that hamper responsiveness to demand of tasks.

Med Segur Trab (Internet) 2014; 60 (236) 497-507

Keywords: Occupational bealth, Adjustments, Disabilities, Workplace, Safety, Risk factor. (Source: $\mathrm{MeSH}, \mathrm{NLM}$ ) 


\section{INTRODUCCIÓN}

El desempeño de actividades laborales por un trabajador que tiene problemas de salud tiene un notable impacto económico para las empresas y serias consecuencias para el propio trabajador.

En las próximas décadas, es previsible que la proporción de profesionales del sector sanitario público con limitaciones de salud o discapacidad aumente por diversos factores, que van desde la inseguridad en el empleo, la presión laboral o el estrés en el lugar de trabajo $^{1}$ hasta otros como el retraso de la edad de jubilación y el envejecimiento de las plantillas de personal ${ }^{2}$. Esto significa que con la edad se incrementará el porcentaje de trabajadores que seguirán activos laboralmente a pesar de que su capacidad de trabajo se verá afectada por sus limitaciones de salud.

La protección de la salud de los trabajadores es objeto de intervención de los poderes públicos, quienes a través de diversas regulaciones han dispuesto que el empresario tenga un deber especial en la protección específica a determinados grupos sensibles o colectivos especialmente vulnerables a ciertos riesgos laborales, dependiendo de su estado biológico y de las condiciones -subjetivas- del trabajador.

La protección de estos trabajadores especialmente sensibles (TES) se basa en adaptar los puestos de trabajo o las condiciones laborales a la persona del trabajador (que no a la inversa), en tutelar su salud, facilitar su integración en el entorno laboral de forma segura, y en procurar que estas personas no pierdan su trabajo en aquellos supuestos en que no se logra adaptar satisfactoriamente el trabajo a la persona.

En el ámbito del Servicio Andaluz de Salud, la adaptación del puesto de trabajo se concreta en las modificaciones o ajustes de las condiciones de trabajo de un puesto específico con el objeto de que un trabajador concreto catalogado previamente como especialmente sensible, pueda desempeñar las funciones propias con garantía de protección eficaz en materia de seguridad y salud en el trabajo ${ }^{3}$. Cuando la naturaleza de las deficiencias funcionales es compleja, la intervención para la adaptación del puesto de trabajo adquiere un papel relevante en la gestión de la prevención de riesgos laborales. En este sentido, el propósito del proceso de adaptación es detectar posibles desajustes que impiden que un trabajador pueda ocupar un determinado puesto evitando que su limitación o discapacidad empeore, que sobrevenga una nueva o se ponga en riesgo la salud de otros trabajadores. Y para ello se persigue conciliar dos componentes: la demanda del trabajo y la aptitud funcional del trabajador para llevar a efecto aquellas tareas que son fundamentales en su puesto de trabajo. En definitiva, la adaptación del puesto de trabajo fomenta acciones de mejora de las condiciones de trabajo, cumpliendo así con una función esencialmente preventiva.

Es escasa la información disponible sobre la prevalencia y naturaleza de las adaptaciones de puestos de trabajo para TES. Estas carencias se traducen en un desconocimiento de la efectividad de la normativa sobre prevención de riesgos laborales cuando se cambian los factores contextuales del trabajo (ambientales y personales), cuando se modifican tareas y/o se reorganiza el trabajo, cuando se cambia de puesto o categoría en función de la aptitud laboral, e incluso en caso extremo, la suspensión o extinción del contrato laboral cuando la adaptación del trabajo a las condiciones de la persona es infructuosa, sin que ello signifique que el empresario actúe unilateralmente en tales circunstancias.

A este respecto, desde hace tiempo se viene cuestionando la efectividad de la normativa en materia de prevención de riesgos laborales porque su cumplimiento se ha dirigido más hacia la justificación documental que a la práctica real de medidas preventivas útiles, llegando a acuñarse el término "prevención de papel»"-6. De ahí que la aplicación efectiva de la legislación que regula la protección del TES plantee algunos interrogantes que pueden ser resueltos a partir del estudio de la casuística de estos colectivos existentes en la realidad de empresas y organizaciones, que aúna a categorías dispares como 
trabajadoras embarazadas y lactantes, personas con discapacidad, menores de edad, así como a trabajadores temporales e inmigrantes. En adición a estos colectivos, la legislación española ${ }^{7}$ estableció un supuesto de aplicación genérica a cualquier trabajador que muestre una especial sensibilidad a determinados riesgos debido a sus características personales o estado biológico o psicofísico, con identificación expresa de tal grupo de riesgo, ya sea con antelación al acceso al puesto o de forma sobrevenida, en los cuales centraremos la presente investigación.

En esta investigación se presenta el estudio de una serie de casos de adaptaciones de las condiciones de trabajo tratados por la Unidad de Prevención de Riesgos Laborales de un Distrito de Atención Primaria de Salud de la provincia de Málaga. El objetivo principal fue conocer cuáles son las características básicas de las adaptaciones de puesto que ayuden a definir el perfil del TES. Un objetivo secundario es avanzar en el conocimiento de los tipos de ajustes y medidas adoptadas en el trabajo para un particular grupo de riesgo en ocupaciones del sector sanitario.

\section{MÉTODO}

Diseño. Observacional. El tipo de estudio consistió en un análisis descriptivo. La unidad básica de investigación fue el informe de restricciones con propuesta de adaptación de puesto de trabajo, definido como: «las modificaciones o ajustes de las condiciones de trabajo de un puesto específico con el objeto de que un trabajador concreto catalogado previamente como especialmente sensible, pueda desempeñar las funciones propias con garantía de protección eficaz en materia de seguridad y salud en el trabajo". Como fuente de información se ha utilizado la base de datos "Siprila" en soporte FileMaker ${ }^{\circledR}$ Pro 6.0 para Windows, que almacena los informes del técnico de prevención sobre adaptación del puesto de trabajo así como las restricciones médicas identificadas como resultado de la vigilancia de la salud del trabajador.

Muestra estudiada. Los participantes fueron todos los trabajadores de los centros de trabajo, cuya cifra alcanza un total de 1361 profesionales, de los que 518 son hombres y 843 mujeres. El análisis se acotó cronológicamente al año 2012, con emplazamiento en los 40 centros sanitarios de un Distrito Sanitario de Atención Primaria de la provincia de Málaga.

Definición de caso. Trabajador especialmente sensible a determinado/s riesgo/s es aquel que por sus características personales, estado biológico o por su discapacidad física, psíquica o sensorial, debidamente reconocida, tiene una susceptibilidad superior al resto de los trabajadores, frente a un determinado riesgo.

Criterios de inclusión. Todas las adaptaciones de puestos trabajo para TES tramitadas por la Unidad de Prevención de Riesgos Laborales, durante el período comprendido entre el 1 de enero del 2012 hasta el 31 de diciembre del 2012. Se excluyeron los grupos de mujeres en situación de embarazo, maternidad o lactancia por requerir de un abordaje específico.

Mediciones principales. A partir del análisis de contenidos y extracción de datos de los informes técnicos de adaptación se creó una base datos para el estudio de las siguientes variables: edad, sexo, categoría profesional, calificación médica del trabajador (apto, apto con restricciones, no apto, en observación), restricción de salud (física, sensorial, cognitiva, comunicativa), número y naturaleza de las adaptaciones (factores ambientales, personales, organizativos y cambios de puesto) y tipología de adaptaciones propuestas. Para la codificación de los campos se ha utilizado fundamentalmente la clasificación contemplada en el procedimiento 15 del sistema de gestión de prevención de riesgos laborales del Servicio Andaluz de Salud ${ }^{3}$. 
Tratamiento estadístico. Se realizó un análisis cuantitativo utilizando el software Microsoft ${ }^{\circledR}$ Excel 2010 v.14 para el cálculo de medias, ratios, porcentajes y presentaciones gráficas. Para las variables se elaboraron tablas y diagramas de barras.

\section{RESULTADOS}

El grupo de casos estuvo formado por 46 trabajadores declarados especialmente sensibles a los que se les reconoció alguna limitación de salud y/o discapacidad durante el año 2012. Tras pasar el reconocimiento médico, 37 de estos trabajadores fueron calificados como "apto con restricciones" cuando su estado psicofísico no respondió a las demandas de su puesto de trabajo, mientras que 9 fueron calificados como «en observación" cuando las conclusiones del estudio del facultativo especialista no fueron definitivas, a la espera de pruebas complementarias y/o interconsultas.

La edad media se situó en los 54 años (SD:6; rango:37-65), sin encontrar diferencias estadísticamente significativas por sexo $(t=2,539$ p-valor=0,207 IC=99\%). El 67,4\% ( $n=31)$ fueron mujeres; aunque la distribución de casos con relación al total de la plantilla mantiene una proporción similar en ambos géneros (3,1-3,6\%). El grupo de edad que concentró mayor número de trabajadores con necesidad de ajustes en sus condiciones de trabajo fue el comprendido por personas del tramo 51-60 años, con un 71,7 \% de las intervenciones realizadas en el período estudiado (figura 1). En términos relativos al total de la plantilla de personal, el colectivo con edades entre 31-40 años reportó 1,5 veces más trabajadores con adaptaciones que el de 51-60 años. La tabla 1 muestra la descripción de las características socio-laborales de los TES.

Figura 1. Distribución por edad y sexo de trabajadores especialmente sensibles

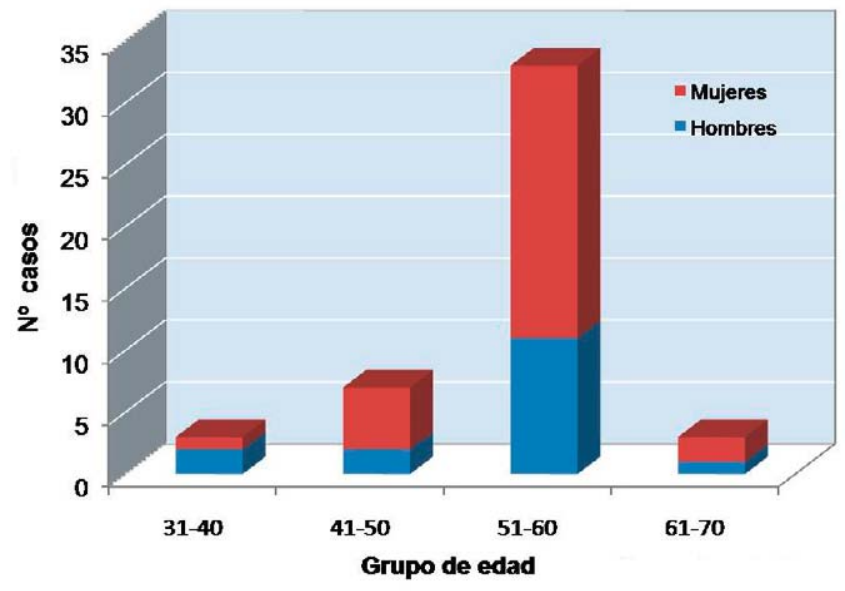

Tabla 1. Características socio-laborales de la población de estudio

\begin{tabular}{lc}
\hline \multicolumn{1}{c}{ Variable } & N \\
\hline Edad media (SD) & $54(6,10)$ \\
Sexo Femenino (\%) & $32(69,6)$ \\
Estudios no universitarios (\%) & $14(30,4)$ \\
Personal Estatutario fijo (\%) & $41(89,1)$ \\
Días por semana & 6 \\
Horas por semana & 37,5 \\
Turno diurno (horas) & 1593 \\
Turno nocturno (horas) & 1460 \\
Turno rotatorio (horas) & 1507 \\
\hline
\end{tabular}


Se identificaron tres categorías generales de adaptaciones: 19 trabajadores recibieron adecuaciones técnicas del puesto focalizadas en el equipamiento y diseño del entorno del puesto y/o lugar de trabajo; 3 lo fueron en cambios de puesto de trabajo dentro de la misma categoría laboral; y en 24 se modificaron las funciones y/o tareas centradas en aspectos organizativos del trabajo y en cambios en la forma de desempeñar aquéllas por el trabajador. La tabla 2 presenta la naturaleza de las medidas de adaptación realizadas para los trabajadores en función del grado de minusvalía reconocido. Atendiendo al promedio de edad de la plantilla de empleados públicos, una parte relativamente pequeña (34 por cada mil trabajadores) comunicó la necesidad de algún ajuste en su ambiente y/o condiciones de trabajo, sobre todo si se tiene en cuenta que más de un tercio $(n=16)$ de los TES tienen reconocido algún grado de discapacidad, principalmente de tipo moderado.

Tabla 2. Naturaleza de las adaptaciones de puesto realizadas y grado de minusvalía reconocido

\begin{tabular}{lccc}
\hline \multicolumn{1}{c}{ Naturaleza de la adaptación } & N & Grado minusvalía (\%) & N \\
\hline Modificación de tareas & 38 & 0 & 30 \\
Reorganización del trabajo & 15 & $1-24$ & 1 \\
Dotación/modificación equipos & 11 & $25-49$ & 11 \\
Diseño del entorno del puesto & 9 & $50-70$ & 3 \\
& & 75 & 1 \\
\hline
\end{tabular}

Las condiciones de salud en los TES fueron principalmente de tipo físico $(n=34)$, y de éstas, la mayoría estuvieron asociadas con el sistema músculo-esquelético (n=29) mientras que las menos habituales fueron el sistema respiratorio y el estado inmunológico del trabajador (tabla 3). Aproximadamente una de cada cuatro adaptaciones fueron debidas a limitaciones de las capacidades sensoriales, mientras que los trabajadores con problemas psicosociales presentaron una probabilidad próxima al $40 \%$ de recibir ajustes en sus condiciones de trabajo frente a otro tipo de discapacidad.

Tabla 3. Principales condiciones de salud que provocan limitaciones laborales

\begin{tabular}{lc}
\hline \multicolumn{1}{c}{ Sistema/Aparato } & N \\
\hline Osteomuscular & 29 \\
Psicológico-emocional & 18 \\
Neurológico-sensorial & 11 \\
Cardiovascular & 7 \\
Endocrinológico & 3 \\
Digestivo & 2 \\
Genitourinario & 2 \\
Respiratorio & 1 \\
Inmunológico & 1 \\
\hline
\end{tabular}

Se han identificado diez categorías profesionales de trabajadores clasificados como especialmente sensibles. El grupo profesional que demandó más adaptaciones fue el de medicina familiar y comunitaria $(n=17)$, casi el doble que el de auxiliar administrativo y enfermería (figura 2). Se estimó un promedio de 3,2 tipos de ajustes por cada trabajador (tabla 4). Los ajustes más comunes entre los médicos fueron la adopción de micropausas periódicas, alternancia de tareas y la racionalización del tiempo de trabajo con el propósito de conciliar el ritmo de trabajo conforme a las aptitudes individuales. 
Figura 2. Categoría laboral de trabajadores especialmente sensibles

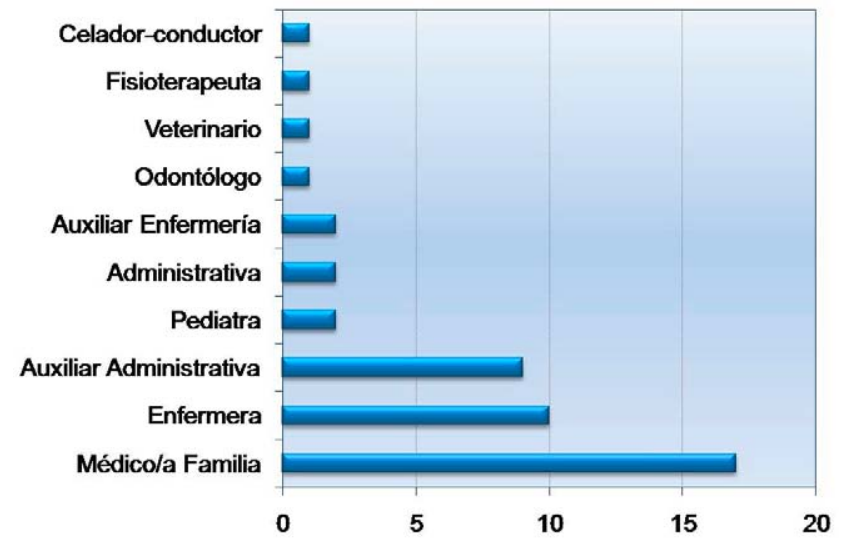

Tabla 4. N. ${ }^{\circ}$ de medidas realizadas por categoría profesional según tipo de adaptación agrupada por sexo y edad

\begin{tabular}{|c|c|c|c|c|c|c|c|c|c|}
\hline \multirow{2}{*}{ Categoría profesional } & \multirow{2}{*}{$\begin{array}{l}\text { Tipo adaptación } \\
\text { (Material/ } \\
\text { Inmaterial) }\end{array}$} & \multicolumn{4}{|c|}{$\begin{array}{l}\text { MASCULINO } \\
\text { Grupo de edad }\end{array}$} & \multicolumn{4}{|c|}{$\begin{array}{c}\text { FEMENINO } \\
\text { Grupo de edad }\end{array}$} \\
\hline & & $31-40$ & $41-50$ & $51-60$ & $61-70$ & $31-40$ & $41-50$ & $51-60$ & $61-70$ \\
\hline \multirow[t]{2}{*}{ Médico/a de familia } & M & & & 3 & & & 4 & 4 & \\
\hline & I & & & 19 & & & 3 & 32 & 3 \\
\hline \multirow[t]{2}{*}{ Enfermera } & M & & & & & 1 & & 4 & \\
\hline & I & 4 & & 9 & & 5 & & 6 & \\
\hline \multirow[t]{2}{*}{ Auxiliar administrativa } & M & & & & & & 1 & & \\
\hline & I & & 1 & & & & 7 & 20 & \\
\hline \multirow[t]{2}{*}{ Pediatra } & M & & & & & & & & 2 \\
\hline & I & & & & & & & 2 & 1 \\
\hline \multirow[t]{2}{*}{ Administrativa } & M & & & & & & & 1 & \\
\hline & $\mathrm{I}$ & & & 1 & & & & 3 & \\
\hline \multirow[t]{2}{*}{ Auxiliar enfermería } & M & & & & & & & & \\
\hline & $\mathrm{I}$ & & & & & & & 4 & \\
\hline \multirow[t]{2}{*}{ Odontólogo } & M & & & & & & & & \\
\hline & I & & & & 1 & & & & \\
\hline \multirow[t]{2}{*}{ Fisioterapeuta } & M & & & & & & 1 & & \\
\hline & $\mathrm{I}$ & & & & & & 1 & & \\
\hline \multirow[t]{2}{*}{ Veterinario } & M & & & & & & & & \\
\hline & I & & & 1 & & & & & \\
\hline \multirow[t]{2}{*}{ Celador-conductor } & M & & & & & & & & \\
\hline & I & & 4 & & & & & & \\
\hline
\end{tabular}

En 25 trabajadores estudiados se produjo falta de correspondencia en parte de las restricciones laborales valoradas por el área de vigilancia de la salud y la evaluación de riesgos laborales realizada por los técnicos de prevención para adoptar medidas armonizadas de adaptación del puesto de trabajo.

Las propuestas de adaptación más frecuentes estaban relacionadas con medidas para superar las limitaciones en la actividad laboral, introduciendo modificaciones en la manera que tiene el trabajador de llevar a cabo sus tareas al efecto de alcanzar un acoplamiento razonable trabajo-trabajador (tabla 5). En este sentido la mayoría de las veces se acordó favorecer la alternancia de tareas y la realización de micropausas de 10 
minutos cada dos horas de trabajo continuado $(n=17)$. La limitación funcional más persistente fue la manipulación manual de cargas $(n=15)$, entendida como una operación de transporte o sujeción de una carga por parte del trabajador, que por sus características o condiciones ergonómicas inadecuadas entrañe riesgos, particularmente dorsolumbares. A este respecto, la adaptación más común fue limitar el peso de la carga que debe manipular el trabajador a menos de $3 \mathrm{Kg}$ así como cumplir con normas básicas de higiene postural para el ejercicio de la actividad laboral en condiciones seguras. En cuanto a las medidas de reorganización del trabajo, la supresión de tareas no esenciales del puesto o la modificación de la jornada, turno u horario estuvieron presentes en 15 casos. El tiempo invertido por el trabajador para realizar sus tareas también fue motivo de adecuación $(n=11)$ admitiendo la posibilidad de disponer de autonomía en la distribución y ejecución de sus cometidos. Las dos medidas de ajuste menos habituales consistieron en la incorporación de una ayuda técnica un trabajador con deficiencia visual y la dotación de un equipo de protección individual para la administración de medicamentos citostáticos.

Tabla 5. Listado de medidas de adaptación del puesto de trabajo realizadas

\begin{tabular}{|c|c|}
\hline Ajustes en las condiciones de trabajo & $\mathbf{N}$ \\
\hline Micropausas periódicas de descanso & 17 \\
\hline Alternancia de tareas & 17 \\
\hline Limitar peso en el manejo manual de cargas & 15 \\
\hline Incorporar medidas de higiene postural & 15 \\
\hline Formación específica & 12 \\
\hline Suprimir tareas (urgencias, visitas domiciliarias, conducir, administrar citostáticos...) & 12 \\
\hline Modular ritmo de trabajo & 11 \\
\hline Factor ambiental (control luminosidad) & 5 \\
\hline Ordenación espacios/mobiliario trabajo & 4 \\
\hline Reducir/quitar jornada complementaria & 4 \\
\hline Dotar asiento/camilla ergonómica & 3 \\
\hline Limitar esfuerzos con pacientes & 3 \\
\hline Evitar sobreesfuerzos físicos & 3 \\
\hline Mejorar distribución de la carga laboral & 3 \\
\hline Sustituir pantalla visualización de datos & 3 \\
\hline Adquirir esfingomanómetro accion. no manual & 2 \\
\hline Cambio de lugar de trabajo (no ambulancia) & 2 \\
\hline Cambio turno de trabajo diurno-nocturno & 2 \\
\hline Organizar tiempo de trabajo & 2 \\
\hline Sustitución tareas de alta demanda & 2 \\
\hline Modificar horario de trabajo mañana-tarde & 1 \\
\hline Utilizar procedimiento de agentes biológicos & 1 \\
\hline Reposo de comidas & 1 \\
\hline Programar visitas domiciliarias & 1 \\
\hline Autocontrol de la voz & 1 \\
\hline Evitar carga dorsolumbar de mochilas & 1 \\
\hline Adquirir ayuda técnica deficiencia visual & 1 \\
\hline Proveer EPI para administrar citostáticos & 1 \\
\hline
\end{tabular}

Las intervenciones en el diseño del entorno de trabajo afectaron a 19 trabajadores, principalmente mediante la dotación o sustitución de equipos o aparatos, regulando la exposición a parámetros ambientales (luz natural-artificial) así como adecuando espacios y mobiliario del lugar o puesto de trabajo. 


\section{DISCUSIÓN}

El análisis de datos posibilitó describir el perfil típico del TES de un centro de salud como el de una mujer de 54 años, de profesión médica, que debido a desórdenes músculoesqueléticos se le asignan micropausas de descanso periódico junto a la posibilidad de modular con autonomía su ritmo de trabajo acorde a la capacidad individual.

El proceso natural de envejecimiento es uno de los aspectos humanos reconocidos ${ }^{8-9}$ que juega un importante papel en las adaptaciones del puesto debido a la aparición de deficiencias y limitaciones funcionales ${ }^{10}$. Sin duda la edad fue un factor contribuyente a una mayor susceptibilidad a ciertos riesgos laborales, puesto que mayoritariamente los trabajadores del tramo 51-60 años tuvieron que enfrentarse a problemas en el diseño del entorno y contenidos de su trabajo, requiriendo de ajustes en su puesto.

Aunque algunos investigadores han encontrado diferencias entre las distintas condiciones de salud y sus efectos en la capacidad de hombres y mujeres, por ejemplo, una mayor incidencia del síndrome ansioso-depresivo en mujeres ${ }^{11}$, globalmente nuestros resultados indican que los ajustes recibidos en ambos sexos fueron similares, sin apreciar diferencias llamativas en cuanto a determinado tipo de factor de riesgo.

Los trabajadores que demandaron más ajustes y cambios en el trabajo contaban con estudios universitarios, lo que significa que una formación más cualificada no implica una mejor adaptación al puesto de trabajo. La complejidad de las tareas, la pérdida de autonomía para realizarlas, el nivel de atención requerido y el sobreesfuerzo intelectual exigido por el tipo de trabajo en estos profesionales son factores que llegan a superar los recursos del individuo para controlar situaciones de estrés laboral ${ }^{12}$, y que derivaron en solicitudes de intervenciones preventivas en el puesto de trabajo.

Con esta investigación se han documentado los tipos de medidas de adaptación que recibieron un conjunto de trabajadores del sector sanitario por su especial vulnerabilidad a ciertos riesgos laborales. Los hallazgos indicaron que, exceptuando un caso, en todos los TES se implantaron medidas de tipo inmaterial, esto es, basadas en cambios en la forma de ejecutar las tareas o bien en aspectos de tipo organizativo, como la supresión de partes no esenciales del trabajo o la modificación de horario, turno o jornada. Los ajustes materiales se produjeron en cuatro de cada diez trabajadores, lo que sugiere una mayor dificultad para dotar equipamientos o proveer ayudas técnicas que superen las barreras asociadas con el estado biológico del individuo. Por el contrario, los ajustes organizativos suelen ser más asequibles, corroborando los resultados de otros autores ${ }^{13}$.

No obstante, los beneficios de las adaptaciones de puestos que se han documentado en la literatura científica indican una evidencia moderada en la disminución del absentismo laboral $^{14-16}$ y una mejora en el rendimiento del profesional ${ }^{17-18}$. Sin embargo, esto no justifica que estas medidas de intervención sean efectivas para mejorar la salud del individuo $^{19}$. En parte esto se explica porque las adaptaciones se orientan para eliminar obstáculos que interfieren en el trabajo de la persona, en lugar de centrarse en la mejora de los síntomas de un problema de salud. Incluso medidas como la formación, presentan evidencias débiles y limitadas ${ }^{20}$ en cuanto a su efectividad en la mejora de las condiciones de seguridad y salud. En cualquier caso, la participación en actividades formativas específicas no tiene efectos negativos por cuanto son útiles para enseñar técnicas de control de tiempos y ritmos de trabajo, analizar el entorno del puesto, trabajar cooperativamente, resolver problemas y buscar alternativas.

Sorprendentemente, las medidas de ajuste trabajo-persona fueron similares a través de los diferentes tipos de limitaciones de salud y fue llamativo el hecho de que en más de la mitad de casos existiesen criterios discordantes entre las restricciones laborales expresadas por vigilancia de la salud y los ajustes propuestos por los técnicos de prevención. Estas discrepancias se basan en incluir tareas en las restricciones médicas que son ajenas al resultado de la evaluación de riesgos, incluso limitaciones de tareas concebidas de forma imprecisa y contradictoria. Los principales signos de estas 
incoherencias fueron equiparar la actividad de «movilización de pacientes» con una ayuda puntual en el posicionamiento; igualar el concepto de manipulación de cargas a un acto residual de manejo, asimilando lo excepcional con lo intolerable; apreciar movimientos repetitivos cuando los ciclos de trabajo son dispares; restricciones de turnos, tareas o servicios (ej. Urgencias) no asignados al trabajador o incluso se consideró como restricción realizar descansos reconocidos legalmente. Estos hallazgos aportan razones para sospechar que en ciertas ocasiones se instrumentaliza el procedimiento de adaptación de puesto con la pretensión de conseguir algún beneficio, mejoras laborales al margen de la condición de salud o para hacer efectivos determinados derechos (exención voluntaria de guardias por razón de edad, libranza de guardias, descanso entre jornadas, descanso semanal de 36 horas ininterrumpidas, conciliación de la vida laboral-familiar). Adicionalmente, cuando las restricciones médicas se expresaron en términos de «evitar/ no realizar» tareas o "quitar/reducir» turnos o jornadas, sin pormenorizar cuál es la condición psicofísica del trabajador, se corre el peligro de desnaturalizar la esencia de este procedimiento de gestión y malograr el objetivo de incorporar medidas de adaptación con base en un estudio técnico.

La conclusión que pueden obtenerse de este estudio es que pueden llevarse a cabo una amplia variedad de medidas de ajustes y modificaciones de las condiciones de trabajo con el fin de gestionar problemas que interfieren en la capacidad de respuesta debido a las exigencias de las tareas del puesto. Ahora bien, para valorar la efectividad de las medidas implantadas y su traducción en resultados positivos en seguridad y salud, se precisará realizar ampliar las investigaciones.

\section{Limitaciones del estudio}

Los datos están basados principalmente en trabajadores que deciden iniciar voluntariamente el procedimiento de adaptación de puestos, subestimando a otras personas que conscientes o no de limitaciones de salud optan por no utilizar esta vía por diversas causas relacionadas con la seguridad en el puesto de trabajo, insatisfacción laboral, infravaloración del problema de salud, entre otros.

Este estudio descriptivo no permite estimar el coste-efectividad de las adaptaciones realizadas y su impacto en salud. Pero son cuestiones que necesitan de una respuesta para valorar si la aplicación práctica de las regulaciones sobre seguridad y salud en el trabajo ofrece resultados positivos. Por ello, una investigación más amplia puede servir para comprobar si los cambios en el contenido y entorno de trabajo repercuten favorablemente en la salud y calidad de vida del trabajador.

\section{REFERENCIAS BIBLIOGRÁFICAS}

1. OECD. Sick on the job? myths and realities about mental health and work. OECD publishing; 2012.

2. González López-Valcárcel B, Barber Pérez P. Oferta y necesidad de médicos especialistas en España (2006-2030). Grupo de investigación en Economía de la Salud, Universidad de las Palmas de Gran Canaria; 2007.

3. Servicio Andaluz de Salud. Sistema de Gestión de Prevención de Riesgos Laborales. Procedimiento 15: Adaptación de puestos a trabajadores especialmente sensibles. Sevilla: Consejería de Salud y Bienestar Social, Junta de Andalucía. (actualizado el 10 May 2013) Disponible en: http://www.sas.junta-andalucia. es/principal/documentosacc.asp?pagina=pr_PrevencionRiesgos_Procedimiento

4. Análisis del fórum PRL sobre las dificultades que tienen las empresas en la gestión de la coordinación de actividades empresariales. En: Coordinación de actividades empresariales 2012. Foment del Treball Nacional. 2012: 26-31

5. Monreal de la Iglesia V. Comentarios a la Ley sobre subcontratación en el sector de la construcción. Rev. Dirección y administración de empresas. 2008 (15): 119-143.

6. Prunes Freixa MA. De qué no se es todavía consciente en prevención de riesgos. Gestión práctica de riesgos laborales. 2006 (33):22-29.

7. Ley de Prevención de Riesgos Laborales. L. N. 31/1995 (8 Nov 1995) 
8. Pagán Castaño P, Ferreras Remesal A, Mena del Horno S et al. Adaptación de puestos de trabajo para trabajadores mayores. Guía de buenas prácticas. Instituto de Biomecánica de Valencia. Universidad Politécnica de Valencia; 2011

9. Jiménez-Martín S. Bienestar 4: Salud, mortalidad y participación laboral en edades avanzadas. Apuntes FEDEA. Barcelona: Universitat Pompeu Fabra; 2011.

10. Bjelland MJ, Bruyère SM, von Schrader S, Houtenville AJ, Ruiz-Quintanilla A, Webber DA. Age and Disability Employment Discrimination: Occupational Rehabilitation Implications. J Occup Rehabil. 2010 (20): 456-71.

11. Mansilla Izquierdo F, García Micó JC, Gamero Merino C, Congosto Gonzalo A. Influencia de la insatisfacción laboral en las demandas de cambio de puesto de trabajo por motivos de salud. Med Segur Trab. 2010; 56 (219): 147-157.

12. Comisión Europea. Guía sobre el estrés relacionado con el trabajo. ¿La «sal de la vida» o el «beso de la muerte»? Dirección General de Empleo, Asuntos Sociales e Inclusión. Luxemburgo: Oficina de publicaciones oficiales de las comunidades europeas; 1999.

13. Baanders AN, Andries F, Rijken PM, Dekker J. Work adjustments among the chronically ill. International Journal of Rehabilitation Research. 2001; 24: 7-14.

14. Van Oostrom SH, Driessen MT, de Vet HC, Franche RL, Schonstein E, Loisel P et al. Workplace interventions for preventing work disability. Cochrane Database Syst Rev. 2009 Apr 15;(2):CD006955.

15. Franche RL, Cullen K, Clarke J, Irvin E, Sinclair S, Frank J. Workplace-based return-to-work interventions: a systematic review of the quantitative literature. J Occup Rehabil. 2005 Dec; 15 (4):607-31.

16. Carson R. Reducing cumulative trauma disorders. Use of proper workplace design. AAOHN J. 1994; 42:270-6.

17. Solovieva TI, Dowler DL, Walls RT. Employer benefits from making workplace accommodations. Disabil Health J. 2011 Jan;4(1):39-45.

18. Schartz HA, Hendricks DJ, Blanck P. Workplace accommodations: evidence based outcomes. Work. 2006; 27(4):345-54.

19. Nieuwenhuijsen K, Bültmann U, Neumeyer-Gromen A, Verhoeven AC, Verbeek JHAM, van der FeltzCornelis CM. Interventions to improve occupational health in depressed people. Cochrane Database of Systematic Reviews. 2008, Issue 3.

20. Kennedy CA, Amick BC, Dennerlein JT et al. Systematic review of the role of occupational health and safety interventions in the prevention of upper extremity musculoskeletal symptoms, signs, disorders, injuries, claims and lost time. J Occup Rehabil. $2010 \mathrm{Jun} ; 20(2): 127-62$.

||||||||||||||||||||||||||||||||||||||||||||||||||||||||||||||||||||||||||||||||||||||||||||||||||||||||||||||||||||||||||||||||||||||||||||||||||||||||||||||||||||||||||||||||||||||||||||||||||||||| || 